\title{
Classical Pathology of Sympathetic Ophthalmia Presented in a Unique Case
}

\author{
Shida Chen ${ }^{1}$, Mary E. Aronow ${ }^{2}$, Charles Wang ${ }^{3}$, Defen Shen ${ }^{1}$ and Chi-Chao Chan ${ }^{*}, 1$ \\ ${ }^{1}$ Immunopathology Section, Laboratory of Immunology, National Institutes of Health, Bethesda, MD, USA \\ ${ }^{2}$ Clinical Branch, National Eye Institute, National Institutes of Health, Bethesda, MD, USA \\ ${ }^{3}$ Christiana Care Health System, Newark, DE, USA
}

\begin{abstract}
The ocular pathology of sympathetic ophthalmia is demonstrated in a 10 year-old boy who sustained a penetrating left globe injury and subsequently developed sympathetic ophthalmia in the right eye two months later. Two and a half weeks following extensive surgical repair of the left ruptured globe, he developed endophthalmitis and was treated with oral and topical fortified antibiotics. One month after the initial injury, a progressive corneal ulcer of the left eye led to perforation and the need for emergent corneal transplantation. The surgical specimen revealed fungus, Scedosporium dehoogii. The boy received systemic and topical anti-fungal therapy. Two months following the penetrating globe injury of the left eye, a granulomatous uveitis developed in the right eye. Sympathetic ophthalmia was suspected and the patient began treatment with topical and oral corticosteroids. Given the concern of vision loss secondary to sympathetic ophthalmia in the right eye, as well as poor vision and hypotony in the injured eye, the left eye was enucleated. Microscopically, granulomatous inflammation with giant cells was noted within a cyclitic membrane which filled the anterior and posterior chamber of the left globe. Other classic features including Dalen-Fuchs nodules were identified. Small, choroidal, ill-defined granulomas and relative sparing of the choriocapillaris were present. Molecular analysis did not identify evidence of remaining fungal infection. The pathology findings were consistent with previously described features of sympathetic ophthalmia. The present case is unique in that co-existing fungal infection may have potentiated the risk for developing sympathetic ophthalmia in the fellow eye.
\end{abstract}

Keywords: Infection, injury, pathology, sympathetic ophthalmia.

\section{INTRODUCTION}

Sympathetic ophthalmia, a disease known since the time of Hippocrates, is a rare, bilateral, granulomatous uveitis that follows penetrating ocular trauma or surgical insult to one eye and threatens sight in the fellow eye which develops inflammation. The injured eye is referred to as the exciting eye, while the fellow eye is called the sympathizing eye. Ocular surgical procedures that disrupt the integrity of the uveo-retinal tissues have lately been shown to be among the important risk factors for developing sympathetic ophthalmia, however penetrating ocular trauma also plays a central role in the incidence of the disease [1]. It has been suggested that co-existing infection may increase the immune response due to greater exposure of retinal antigens and ultimately trigger an autoimmune process that leads to sympathetic ophthalmia [2-4]. While there seems to be no overall gender or racial predilection, the higher incidence of ocular trauma in males and children may explain the higher incidence of sympathetic ophthalmia in these groups reported in some series $[5,6]$. Due to the rare nature of this disease, our current understanding of the pathophysiologic mechanisms underlying sympathetic ophthalmia is limited.

*Address correspondence to this author at the 10 Center Dr., 10/10N103, NIH/NEI, Bethesda, MD 20892-1857, USA; Tel: 301-496-0417;

Fax: 301-402-8664; E-mail: chanc@nei.nih.gov
Herein, we describe the classic ocular histopathology of a patient with sympathetic ophthalmia secondary to penetrating globe injury.

\section{CASE REPORT}

This study was approved by the National Eye Institute Institutional Review Board (IRB) for human subjects, and informed consent was obtained from the patient. A 10 yearold Mennonite boy sustained a traumatic perforation of the left globe due to a knife injury. He underwent extensive surgical repair including anterior chamber washout of $80 \%$ hyphema, lensectomy, vitrectomy for vitreous hemorrhage, and endolaser treatment for a posterior exiting wound in the infratemporal macula. Afterwards, he received intravitreal antibiotics including amikacin and vancomycin. Two weeks following the initial injury, he developed endophthalmitis. He underwent a vitreous and anterior chamber tap and was treated with intravitreal ceftazidime and vancomycin. $\mathrm{He}$ was also started on topical antibiotics including hourly besifloxacin and moxifloxacin. Cultures revealed rare fungal elements, but no bacteria. He was therefore started on topical hourly voriconazole and oral voriconazole $100 \mathrm{mg}$ twice daily for 4 weeks. Due to concern for possible co-existing bacterial infection, oral doxycycline was added to his regimen. He developed a progressive corneal ulcer, and given the concern for Pseudomonas, standard topical antibiotics were switched to fortified topical hourly 
ceftazidime and vancomycin. One month after the injury, the corneal ulcer perforated, resulting in the need for emergent corneal transplantation in the left eye. The surgical specimen from the host cornea demonstrated fungal elements. DNA sequencing later revealed Scedosporium dehoogii. Seven weeks after injury, oral voriconazole was discontinued as there were no signs of residual infection. Two months following the penetrating globe injury of the left eye, granulomatous uveitis developed in the fellow, right eye. Sympathetic ophthalmia was suspected and the patient began treatment with topical prednisolone acetate $1 \%$ every 2 hours and oral prednisone $(1.5 \mathrm{mg} / \mathrm{kg} /$ day $)$ which was gradually tapered over the next 3.5 months. The vision in the left eye was light perception only. In the right eye, the keratic precipitates (KPs) on the endothelium and anterior lens capsule and cellular reaction in the anterior chamber did not respond to corticosteroid treatment. The vision in the right eye was 20/20. Ten weeks after the injury, enucleation of the left eye was performed given the concern for vision loss secondary to sympathetic ophthalmia in the right eye, as well as blindness and hypotony in the left eye.

Routine histopathology and immunohistochemistry were performed on the enucleated left globe. Immunohistochemistry was carried out using the avidin-biotin-complex immunoperoxidase technique with the Betazoid DAB Chromogen Kit (Biocare medical, CA, USA). Primary antibodies included CD68 (macrophage and microglia), CD3 (T-lymphocyte), and CD20 (B-lymphocyte) (Dako North America, Inc., Carpinteria, CA, USA). Secondary antibody was biotinylated goat anti-mouse $\operatorname{IgG}(1: 200$; Vector Laboratories, CA, USA). Microdissection was performed manually on uncovered stained glass slides, which were from formalin-fixed, paraffin-embedded tissue cut at 4-6 $\mu \mathrm{m}$. Tissue from an area of observed granulomatous inflammation in the region of the pupillary membrane and cyclitic membrane was microdissected for evaluation of fungal DNA. The microdissected tissue was immediately digested with Proteinase K buffer. Polymerase chain reaction (PCR) followed by gel electrophoresis was used to detect the amplification of fungal DNA. The oligonucleotide primers were synthesized by Integrated DNA Technologies, Inc. (IA, USA). Primers for $18 \mathrm{~S}$ universal rDNA (Forward: 5-ATT GGA GGG CAA GTC TG-3; Reverse: 5-CCG ATC CCT
AGT CGG CAT AG-3) and primers for Scedosporium dehoogii (MSDE1: 5- CGC CCG AAA GGA CGA CGG C3; MSA2: 5-CTA CTC GAC TCG TCG AAG GAG C-3) were used. PCR amplification with 32P labeled primers was performed in a Peltier thermal cycler PTC 200 (M J research, Inc, Watertown, MA, USA) in a $10 \mu$ l-volume Eppendorf tube, containing $2 \mu 1$ of extracted sample DNA, $1 \mu \mathrm{l}$ of $10 \mathrm{x}$ Taq buffer, 1 unit of Taq DNA polymerase, $1 \mu \mathrm{l}$ of $\mathrm{MgCl}_{2}$ $(25 \mathrm{Mm}), 2 \mu \mathrm{l}$ of a four-deoxynucleoside triphosphate mix (10 $\mathrm{mM}$ each), and $2 \mu \mathrm{l}$ of each primer (forward and reverse). The PCR amplification protocol was based on a previous publication with some modifications [7, 8]. For $18 \mathrm{~S}$ universal rDNA primer: an initial five-minute denaturation at $95^{\circ} \mathrm{C}$, followed by 45 cycles of 1 min denaturation at $95^{\circ} \mathrm{C}$, $1 \mathrm{~min}$ annealing at $54.5^{\circ} \mathrm{C}$, and $1 \mathrm{~min}$ extension at $72^{\circ} \mathrm{C}$, final extension step was $5 \mathrm{~min}$ at $72^{\circ} \mathrm{C}$. For Scedosporium dehoogii primers: 5 min denaturation at $95^{\circ} \mathrm{C}$, followed by 35 cycles of $45 \mathrm{~s}$ denaturation at $94^{\circ} \mathrm{C}, 45 \mathrm{~s}$ annealing at $65^{\circ} \mathrm{C}$, and $1 \mathrm{~min}$ extension at $72^{\circ} \mathrm{C}$, final extension step was $10 \mathrm{~min}$ at $72^{\circ} \mathrm{C}$. The amplification products were analyzed by $15 \%$ polyacrylamide gel electrophoresis in TBE buffer. Following gel electrophoresis and overnight autoradiography, the PCR products were visualized using a molecular imager (Bio-Rad Laboratories, CA, USA).

Macroscopically, a large corneal transplant button with corneal sutures was noted (Fig. 1A). There was a thick, proliferative, fibrous membrane in the pupillary area. The retina was totally detached and formed a funnel configuration, admixed with subretinal hemorrhage (Fig. 1B). Microscopically, corneal scar tissue was noted circumferentially along the peripheral corneal margin (Fig. 2A, B). There were Bowman's membrane fragments and thick, dense, fibrous tissue with inflammatory cells and small neovascular lumens present within the corneal scar (Fig. 2C). The anterior chamber was shallow and contained erythrocytes and lymphocytes. The anterior chamber angle was narrow with peripheral anterior synechiae present as well as a dense pupillary membrane (Fig. 2A). Posterior synechiae from the necrotic iris were also adherent to the cyclitic membrane. Granulomatous inflammation containing many CD68+ macrophages and some multi-nucleated giant cells surrounded by lymphocytes $(\mathrm{CD} 3+>\mathrm{CD} 20+$ cells) was present within the pupillary membrane, the cyclitic
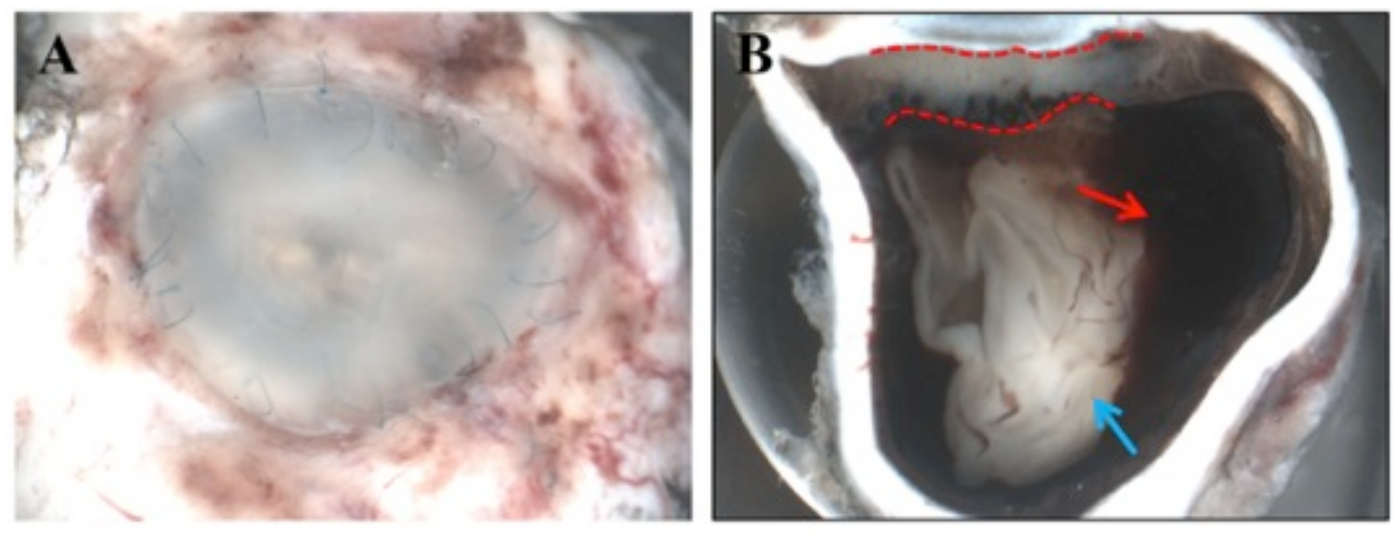

Fig. (1). Macroscopic photographs of the enucleated left eye. (A). There is a large corneal transplant button with corneal sutures. (B) There was a thick, proliferative, fibrous membrane in the pupillary area (dashed line). The retina was totally detached and formed a funnel configuration (blue arrow), admixed with hemorrhage (red arrow). 
A

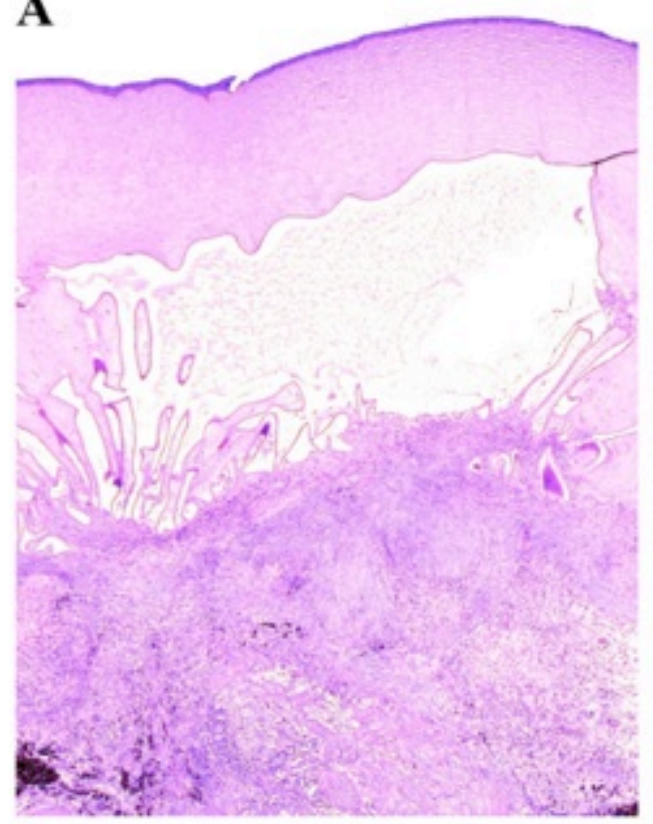

B

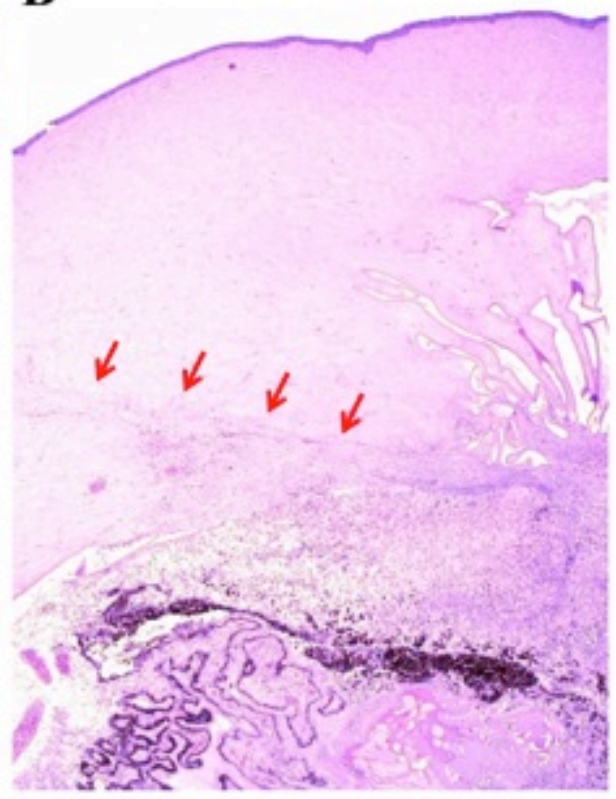

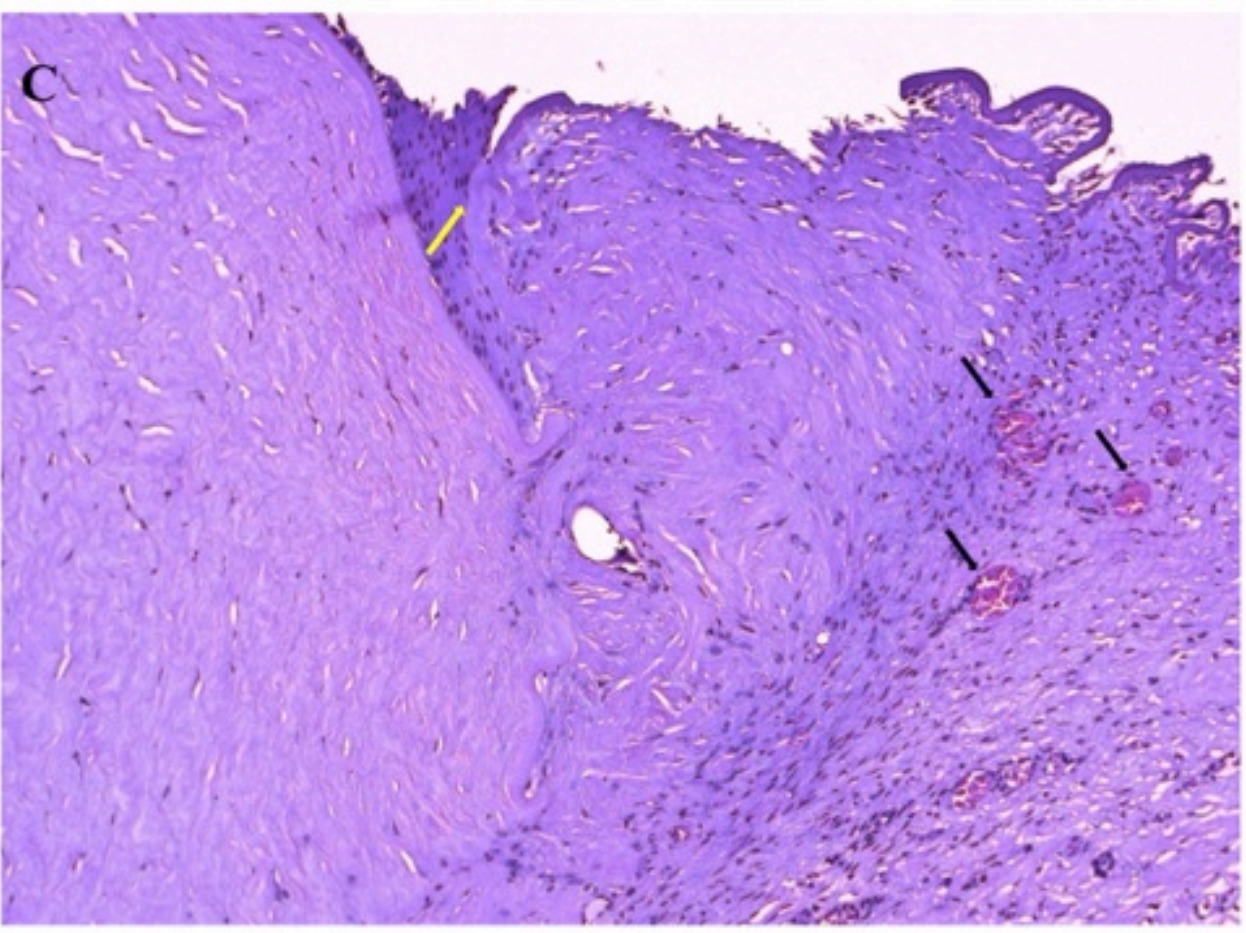

Fig. (2). Photomicrographs of the corneal surgical scar. (A) The anterior chamber angle was narrow with peripheral anterior synechiae, and a dense pupillary membrane. (B) Corneal scar tissue was noted circumferentially along the peripheral corneal margin (red arrow). (C) There were Bowman's membrane fragments (yellow arrow) and thick, dense, fibrous tissue with inflammatory cells and small neovascular lumens (black arrow) present within the corneal scar. (hematoxylin \& eosin, x25 (A \& B), x100, (C)).

membrane, and the anterior vitreous (Fig. 3). There were a few lens capsule fragments and lens cortex remnants within the peripheral cyclitic membrane (Fig. 4A, B). The retina was totally detached in a funnel-shaped configuration, and appeared relatively intact in many areas. Dalen-Fuchs nodules were noted beneath the RPE layer (Fig. 5A, B) and they were composed of predominantly macrophages/epithelioid cells. Small, ill-defined granulomas were also seen in the choroid, along with some CD68+ macrophages, a few CD3+
T lymphocytes, and rare CD20+ B lymphocytes (Fig. 6). The choroid had focal and diffuse monocytic infiltration, and the choroidal vessels were congested in some areas with overall relative sparing of the choriocapillaris (Fig. 7). No fungi were identified with Grocott-Gomori's methenamine silver stain (GMS). The tissues that demonstrated granulomatous inflammation were microdissected for PCR. However, no fungal DNA was detected using an $18 \mathrm{~S}$ primer set or a primer set specific for Scedosporium dehoogii (Fig. 8). 

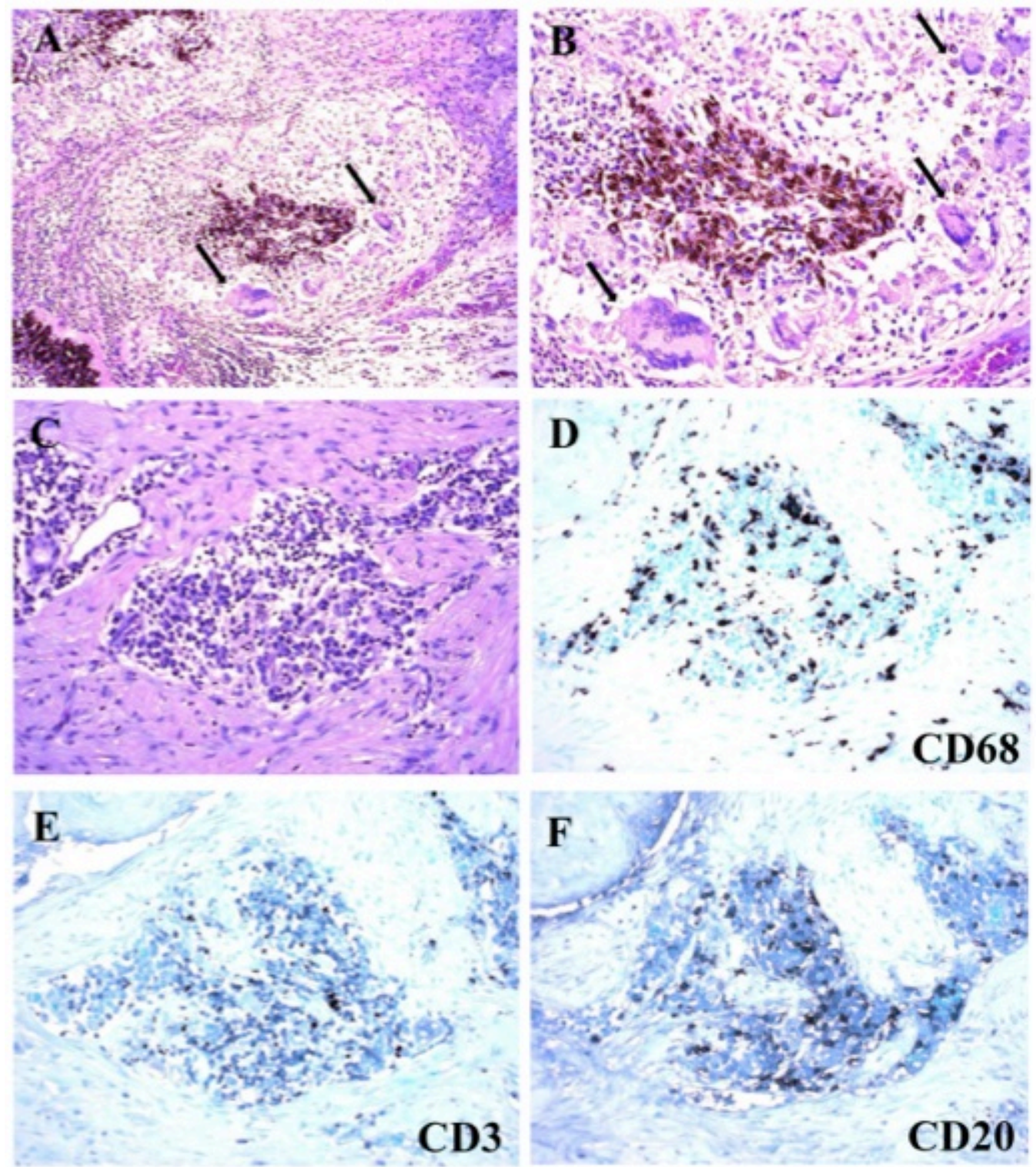

Fig. (3). Photomicrographs of granulomatous inflammation within the pupillary membrane and cyclitic membrane. (A and B) Granuloma in the cyclitic membrane with some multi-nucleated giant cells (black arrow). (C-F) The granulomas in the pupillary membrane contained many CD68+ macrophages surrounded by lymphocytes (CD3+ > CD20+ cells). (A-C, hematoxylin \& eosin, x100 (A), x200 (B-C); D-F, immunohistochemistry staining, $\mathrm{x} 200$ ).
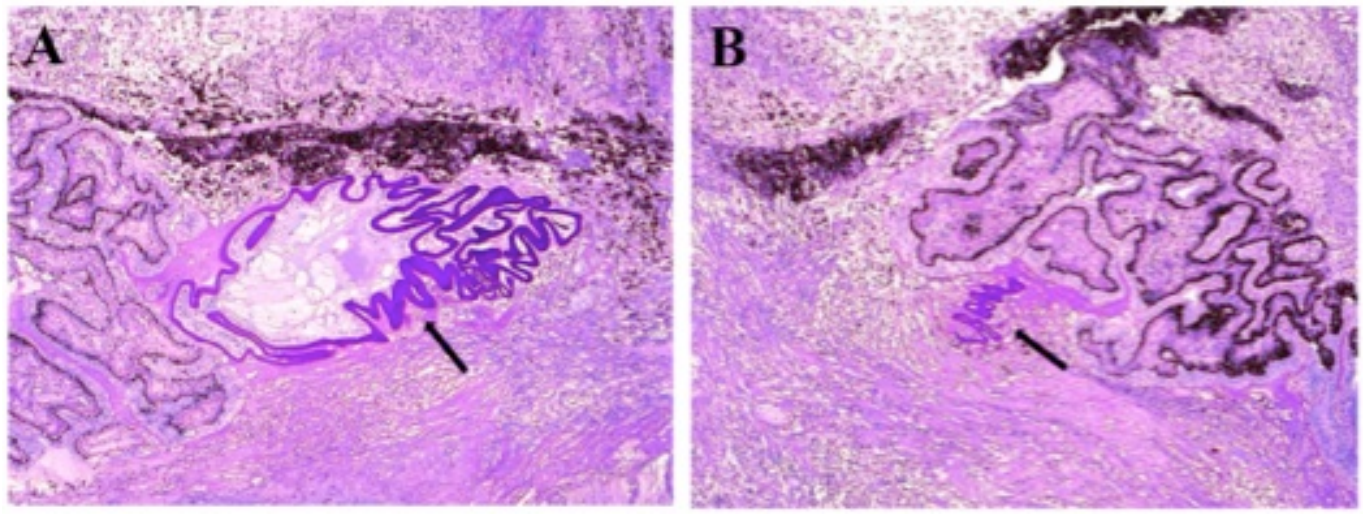

Fig. (4). Photomicrograph of lens capsule fragments and lens cortex. The lens remnants (black arrow) were located within the peripheral cyclitic membrane. (hematoxylin \& eosin, x50). 

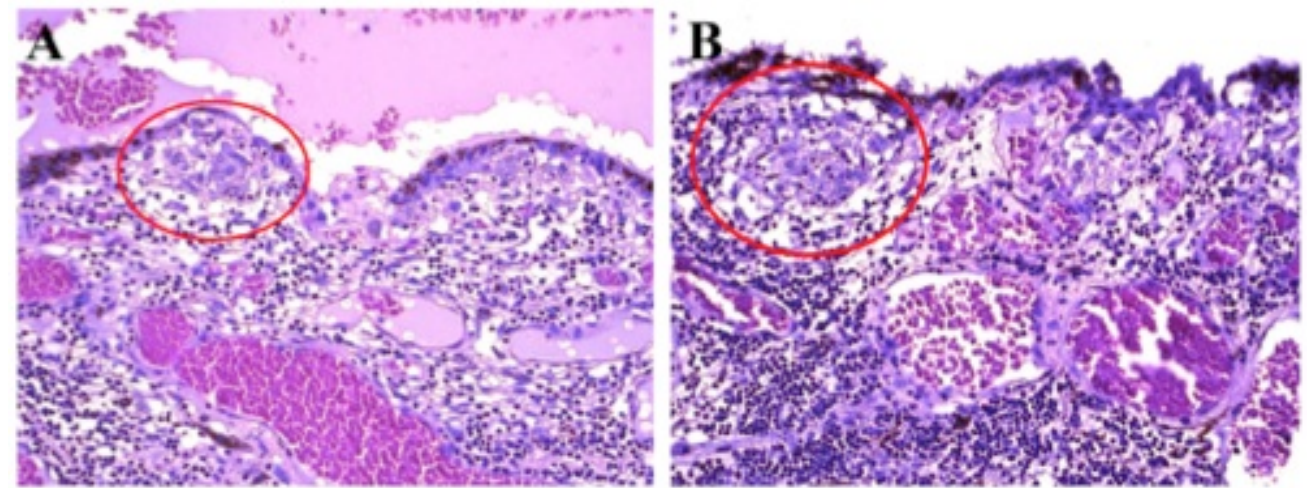

Fig. (5). Photomicrograph of Dalen-Fuchs nodules. Dalen-Fuchs nodules (red circle) were composed of mainly macrophages/epithelioid cells. (hematoxylin \& eosin, x200).
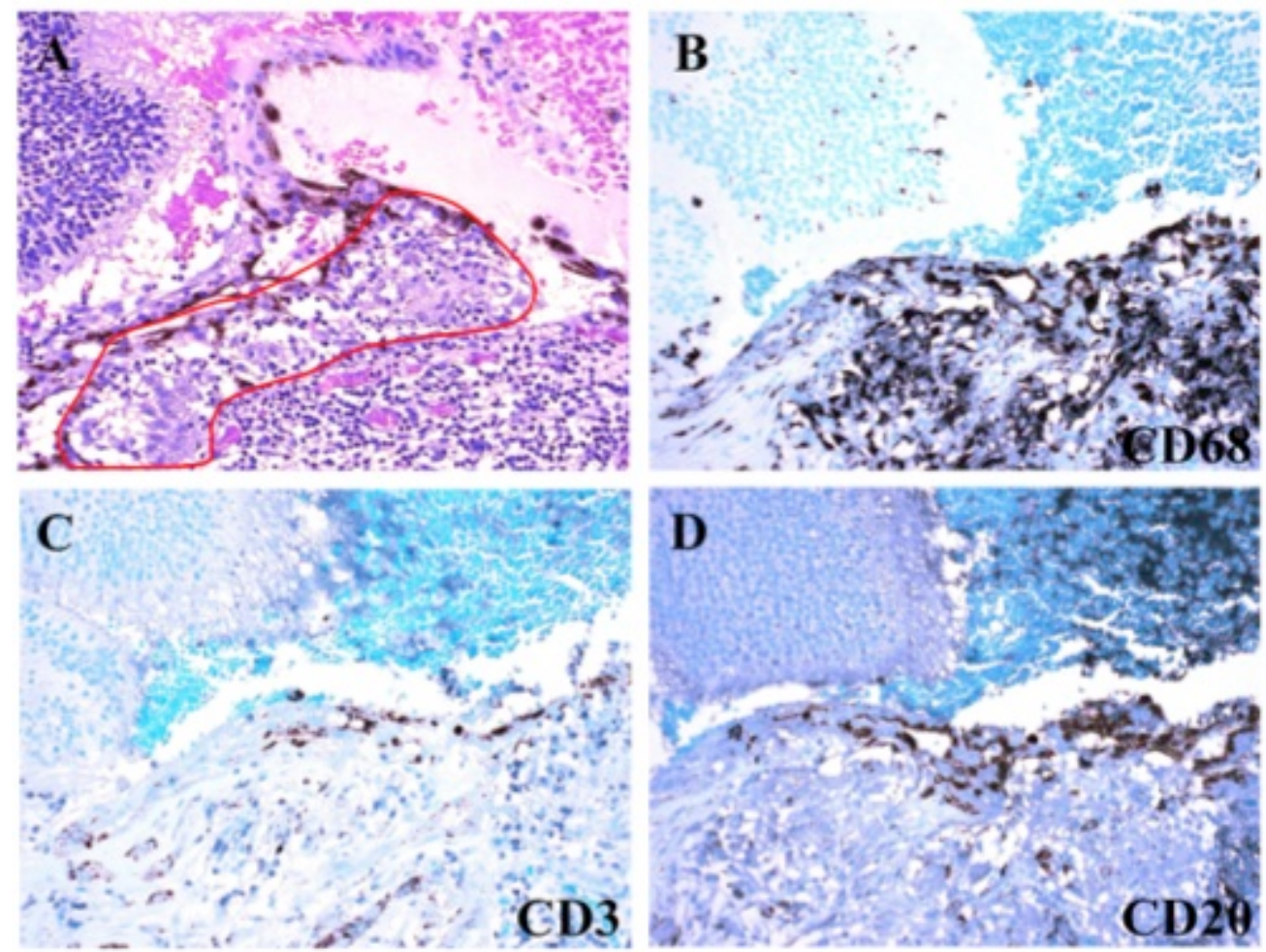

Fig. (6). Photomicrograph of the inflamed choroid. Small and ill-defined granulomas were composed of CD68+ macrophages, a few CD3+ T lymphocytes, and rare CD20+ B lymphocytes. (A: hematoxylin \& eosin, x100; B-D: immunochemistry staining, x200).
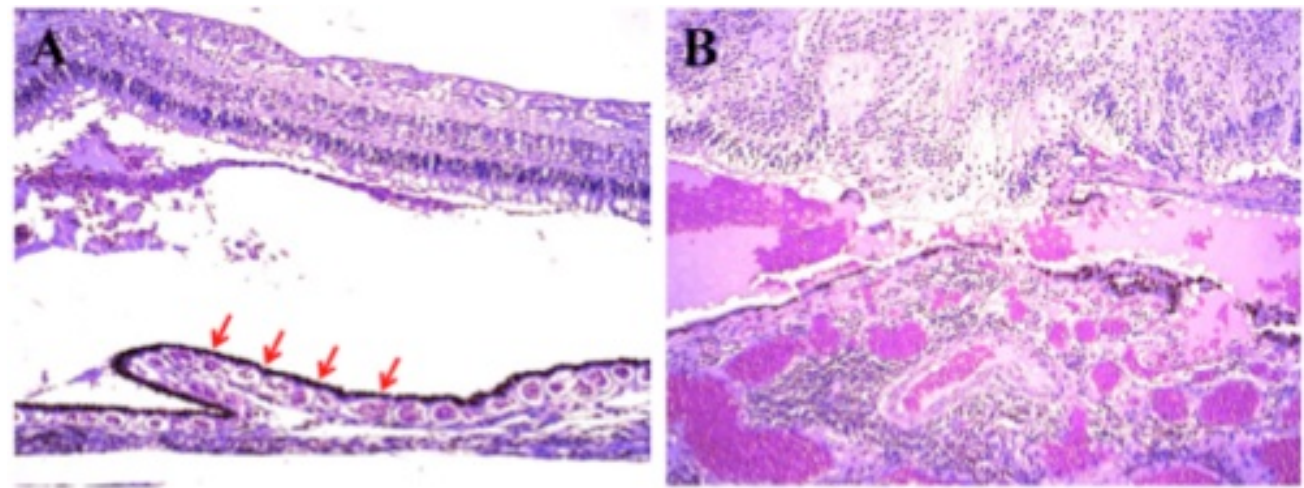

Fig. (7). Photomicrographs of the retina and choroid. (A) The retinal architecture appeared to be relatively normal, and the choroid contained relatively normal choriocapillaris (red arrow) in some areas. (B) The choroid had focal and diffuse monocytic infiltration, and the choroidal vessels were congested in other areas. (hematoxylin \& eosin, $\mathrm{x} 100$ ). 


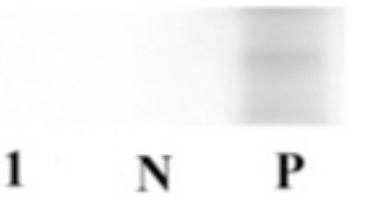

\section{$700 \mathrm{bp}$}

\section{$1 \quad \mathrm{~N} \quad \mathrm{P} \quad 2$}

Fig. (8). PCR products of fungal DNA after gel electrophoresis. (A) Fungal DNA was not detected using an $18 \mathrm{~S}$ primer set in this case. (B) Fungal DNA was not detected using a primer set specific for Scedosporium dehoogii in this case. 1, 2: DNA from the patient; P: positive; $\mathrm{N}$ : negative; bp: base pair.

\section{DISCUSSION}

The present case describes a 10 year-old child who sustained a penetrating knife injury to his left eye and two weeks later developed fungal endophthalmitis. Approximately two months following the injury, the fellow, right eye developed a granulomatous uveitis, and sympathetic ophthalmia was suspected clinically. The patient subsequently underwent enucleation of the injured left eye. The histopathology confirmed the diagnosis of sympathetic ophthalmia.

The histopathology of the patient's eye demonstrated classic features of sympathetic ophthalmia. Widespread uveal granulomatous inflammation was present, which is a hallmark feature and is characterized by focal collection of macrophages and multi-nucleated giant cells surrounded by lymphocytes. Phacoanaphylaxis may also cause granulomatous inflammation and mimic sympathetic ophthalmia, however, phacoanaphylactic uveitis mainly involves the anterior segment with zonal granulomatous inflammation centered around the lens $[3,9]$. In the present case, remnants of the lens capsule and cortex were observed, whereas they were not surrounded by intense inflammatory infiltration and did not induce phacoanaphylactic endophthalmitis. Dalen-Fuchs nodules are a classic feature of sympathetic ophthalmia, and are reported in $25 \%-35 \%$ of cases [10]. The Dalen-Fuchs nodules are predominantly located beneath the RPE layer, and are mainly composed of macrophages [11]. In some cases, when the disease is more advanced, these nodules may also contain depigmented RPE cells or lymphocytes [12, 13]. Previously described cases often report sparing of the retina and choriocapillaries, although one study demonstrated focal involvement of the choriocapillaris in $40 \%$ of cases [14]. In this same series, retinal detachment was observed in $50 \%$ of enucleated eyes, and this was associated with severe uveal inflammation [14].

Although, the exact etiology of sympathetic ophthalmia is not known, it has been hypothesized that altered tolerance to uveal and/or retinal antigens may be involved in the pathogenesis of this disease. One convincing theory suggests that the cell-mediated immune response to antigens from the retinal photoreceptor layer plays an integral role [15]. Implicated retinal antigens include: retinal soluble antigen (S-antigen) [16], rhodopsin [17], and interphotoreceptor retinoid-binding protein [18]. These antigens may induce a cell-mediated immune response. Lymphatics have also been suggested to play a role in the development of sympathetic ophthalmia $[19,20]$. When ocular injury occurs, uveal tissue is exposed to conjunctival lymphatics, and antigens move to the regional lymph nodes, causing a cell-mediated immune response. In a rabbit model, subconjunctival injection of retinal S-antigen in one eye induced a bilateral sympathetic uveitis, however intraocular injection was ineffective in inducing sympathetic disease [20].

In early studies, it was suggested that a purulent eye infection such that occurs with penetrating ocular injury would destroy the uveal tissue and antigens to such an extent that this would prevent the development of sympathetic ophthalmia [10]. However, there are still a few case reports on the occurrence of sympathetic ophthalmia after endophthalmitis $[2,21]$. Buller and associates reported a case of sympathetic ophthalmia following severe fungal keratitis, and hypothesize that chronic fungal infection allowed diffusion of intraocular microorganism antigens and proinflammatory mediators through a disturbed blood-retinal barrier to expose retinal antigens [2]. In one retrospective case series, 4 of 26 patients clinically diagnosed with sympathetic ophthalmia between 2002 and 2004, had concurrent bacterial endophthalmitis, which suggested that purulent endophthalmitis may not prevent the development of sympathetic ophthalmia [3]. The incidence of co-exiting endophthalmitis and sympathetic ophthalmia varies from $1 \%$ to $11 \%$ according to different studies $[3,10,22]$. One theory of pathogenesis suggests that co-exiting infection may increase the risk of development of sympathetic ophthalmia, because ocular infection provokes an immune response involving exposed retinal antigens and incites an autoimmune reaction [2,3]. Experimental animal models have shown some evidence that bacterial products may function as an adjuvant entity which can enhance the uveitogenic potential of uveal and retinal antigens [23]. In our case, the GMS staining and fungal DNA detection studies using PCR in the enucleated eye were negative. This may be due to the fact that after one month of aggressive topical and oral anti-fungal treatment, and there were no signs of residual infection clinically.

Until now, there have been two major modalities employed in the clinical management of sympathetic ophthalmia: surgery (which includes either enucleation or evisceration aimed at prevention) and corticosteroid or immunosuppressive agents for treatment once disease develops. In this case, the injured eye was enucleated as there was concern for further vision loss of the fellow, right eye and no significant clinical improvement after the initiation of topical and oral corticosteroid treatment. There is controversy about the exact time to perform the enucleating surgery for the injured eye. Prompt action is generally recommended, because sympathetic ophthalmia has been reported as early as 5 days after injury and early 
enucleation of the injured eye has been shown to improve the visual prognosis of the sympathizing eye [10], Others question the appropriateness of preventative enucleation based on the fact that the exciting eye may actually retain better vision than the sympathizing eye in the course of the disease. Histologic studies have shown no benefit from enucleation of the exciting eye [24]. While significant controversy still exists regarding evisceration and enucleation, improved surgical techniques have resulted in a recent trend favoring evisceration due to improved cosmetic outcomes [15]. Regarding the importance of concurrent infection, early diagnosis of co-existing mixed infectious and inflammatory processes is important, and the prompt initiation of aggressive antimicrobial treatment as well as systemic corticosteroids may improve the prognosis in such cases [3].

\section{CONCLUSION}

This report demonstrated classic histopathologic features of sympathetic ophthalmia, which occurred secondary to a penetrating globe injury and concurrent fungal infection. This case may improve our understanding of the pathogenesis of sympathetic ophthalmia.

\section{CONFLICT OF INTEREST}

The authors declare no conflict of interest. Informed consent was provided from the patient.

\section{ACKNOWLEDGEMENTS}

The NEI Intramural Program provided funding support.

\section{REFERENCES}

[1] Kilmartin DJ, Dick AD, Forrester JV. Prospective surveillance of sympathetic ophthalmia in the UK and Republic of Ireland. Br J Ophthalmol 2000; 84(3): 259-63.

[2] Buller AJ, Doris JP, Bonshek R, Brahma AK, Jones NP. Sympathetic ophthalmia following severe fungal keratitis. Eye 2006; 20(11): 1306-7.

[3] Rathinam SR, Rao NA. Sympathetic ophthalmia following postoperative bacterial endophthalmitis: a clinicopathologic study. Am J Ophthalmol 2006; 141(3): 498-507.

[4] Sy-Ongkeko JM, Agahan AL, Lopez JS, Dy-Liacco JU. Sympathetic ophthalmia in an infected post-scleral buckling eye. Ann Acad Med Singap 2011; 40(3): 147-8.

[5] Chan CC, Roberge RG, Whitcup SM, Nussenblatt RB. 32 cases of sympathetic ophthalmia: a retrospective study at the National Eye Institute, Bethesda, Md., from 1982 to 1992. Arch Ophthalmol 1995; 113(5): 597-600.

[6] Albert DM, Diaz-Rohena R. A historical review of sympathetic ophthalmia and its epidemiology. Surv Ophthalmol 1989; 34(1): $1-14$.
[7] Kim E, Chidambaram JD, Srinivasan M, et al. Prospective comparison of microbial culture and polymerase chain reaction in the diagnosis of corneal ulcer. Am J Ophthalmol 2008; 146(5): 714-23, 723 e 711.

[8] Harun A, Blyth CC, Gilgado F, Middleton P, Chen SC, Meyer W. Development and validation of a multiplex PCR for detection of Scedosporium spp. in respiratory tract specimens from patients with cystic fibrosis. J Clin Microbiol 2011; 49(4): 1508-12.

[9] Easom H, Zimmerman LE. Sympathetic ophthalmia and bilateral phacoanaphylaxis. a clinicopathologic correlation of the sympathogenic and sympathizing eyes. Arch Ophthalmol 1964; 72: 9-15.

[10] Lubin JR, Albert DM, Weinstein M. Sixty-five years of sympathetic ophthalmia. A clinicopathologic review of 105 cases (1913-1978). Ophthalmology 1980; 7(2): 109-21.

[11] Chan CC, BenEzra D, Hsu SM, Palestine AG, Nussenblatt RB. Granulomas in sympathetic ophthalmia and sarcoidosis. Immunohistochemical study. Arch Ophthalmol 1985; 103(2): 198202.

[12] Reynard M, Riffenburgh RS, Minckler DS. Morphological variation of Dalen-Fuchs nodules in sympathetic ophthalmia. Br J Ophthalmol 1985; 69(3): 197-201

[13] Jakobiec FA, Marboe CC, Knowles DM, et al. Human sympathetic ophthalmia. An analysis of the inflammatory infiltrate by hybridoma-monoclonal antibodies, immunochemistry, and correlative electron microscopy. Ophthalmology 1983; 90(1): 76-95.

[14] Croxatto JO, Rao NA, McLean IW, Marak GE. A typical histopathologic features in sympathetic ophthalmia. A study of a hundred cases. Int Ophthalmol 1982; 4(3): 129-35.

[15] Chu XK, Chan CC. Sympathetic ophthalmia: to the twenty-first century and beyond. J Ophthalmic Inflamm Infect 2013; 3(1): 49.

[16] de Kozak Y, Sakai J, Thillaye B, Faure JP. S-antigen-induced experimental autoimmune uveoretinitis in rats. Current Eye Res 1981; 1(6): 327-37.

[17] Schalken JJ, Winkens HJ, Van Vugt AH, De Grip WJ, Broekhuyse RM. Rhodopsin-induced experimental autoimmune uveoretinitis in monkeys. Br J Ophthalmol 1989; 73(3): 168-72.

[18] Gery I, Wiggert B, Redmond TM, et al. Uveoretinitis and pinealitis induced by immunization with interphotoreceptor retinoid-binding protein. Invest Ophthalmol Vis Sci 1986; 27(8): 1296-300.

[19] Chaithanyaa N, Devireddy SK, Kishore Kumar RV, Gali RS, Aneja V. Sympathetic ophthalmia: a review of literature. Oral Surg Oral Med Oral Pathol Oral Radiol 2012; 113(2): 172-6.

[20] Rao NA, Robin J, Hartmann D, Sweeney JA, Marak GE, Jr. The role of the penetrating wound in the development of sympathetic ophthalmia experimental observations. Arch Ophthalmol 1983; 101(1): 102-4.

[21] Blum-Hareuveni T, Rehany U, Rumelt S. Devastating endophthalmitis following penetrating ocular injury during night sleep from orthodontic headgear: case report and literature review. Graef Arch Clin Exp Opthalmol 2006; 244(2): 253-8.

[22] Luo Y, Wang Z, Lin X, Hu S. Sympathetic ophthalmia caused by ocular penetration with endophthalmitis. Yan ke xue bao 2003; 19(2): 75-8.

[23] Rao NA, Wong VG. Aetiology of sympathetic ophthalmitis. Trans Ophthalmol Soc UK 1981; 101(Pt 3)(3): 357-60.

[24] Winter FC. Sympathetic uveitis; a clinical and pathologic study of the visual result. Am J Ophthalmol 1955; 39(3): 340-7. 